\title{
Research on the Reliability of the Operating Performance of the Relief Valve of Nuclear Power Plant under Seismic Conditions
}

\author{
Shang Huazheng ${ }^{1}$, Yu Xinhai*1 \\ ${ }^{1}$ East China University of Science and Technology, Shanghai,China
}

\begin{abstract}
Nowadays, environmental and energy problems are becoming increasingly prominent. Nuclear power plants are an important means to solve these two problems. Therefore, research on the reliability of nuclear power plants is closely related to environmental and energy issues. The reliability research of nuclear safety valves has always been the top priority in the reliability research of nuclear power plants, so the reliability of the operation performance of such relief valves must be considered under seismic conditions. This article borrows ANSYS to carry out fluid-solid coupling simulation of relief valves, including simulations under seismic conditions and non-seismic conditions, and explores changes during opening and closing under various conditions, including the opening height, pressure and velocity in internal flow field ,inlet pressure, solid stress and solid strain. Finally, a test comparison shows that the effect of longitudinal seismic load on the return pressure of the relief valve can be ignored.
\end{abstract}

\section{Introduction}

Nowadays, environmental and energy problems are becoming increasingly prominent, and nuclear power plants are an important means to solve these two problems. Therefore, research on the reliability of nuclear power plants is closely related to environmental and energy issues. The reliability research of nuclear safety valves has always been the top priority in the reliability research of nuclear power plants, so the reliability of the operation performance of such safety valves must be considered under seismic conditions. The relief valve is an automatic valve. It can discharge a rated amount of fluid by using the pressure of the medium, so as to prevent boilers, pressure vessels and other equipment from being damaged by overpressure. It can ensure the normal operation of the equipment and the safety of personnel. Therefore, the reliability of the relief valve must be guaranteed, even under seismic conditions. At present, predecessors have done a lot of research in related fields.

Guo [1] studied the flow field characteristics of the pressure regulating valve and the spool movement law in the gearbox hydraulic control system, and performed fluid-solid coupling simulation on the flow field and spool of the pressure regulating valve, and obtained the spool's state of stress and deformation at different openings.Deng et al. [2] analyzed the clogging failure caused by viscous heating fluid in the spool valve through fluid-structure coupling simulation. Zhou [3] analyzed the solid domain and fluid domain of the spring tube of the electro-hydraulic servo valve by setting up a two-way fluid-solid coupling, and obtained the stress state of the spring tube at different times. Sang-Uk Han et al. [4] studied the dynamic characteristics of butterfly valves under seismic conditions, and used CQC method and $2 \%$ damping ratio design response spectrum for seismic evaluation. Zhao et al. [5] used fluid-solid coupling to study the closing process of a spherical check valve and obtained values such as stress and strain. Koji Nishino et al. [6] studied the operability of the main steam relief valve used in boiling water reactor nuclear power plants under high acceleration seismic loads. Chen et al. [7] used three-dimensional fluid-structure coupling to analyze the opening and closing process of the reed valve of a miniature compressor. Liu et al. [8] used CFX to numerically simulate two three-eccentric butterfly valves and verified the flow resistance coefficient

Many flow field analysis and unidirectional fluidsoild coupling analysis in the simulation of relief valves in existing studies are studied while there are few studies on two-way fluid-solid coupling, so this paper uses twoway fluid-solid coupling to analyze the reliability of the relief valve in seismic conditions that used in nuclear power plant.

\section{Model Establishment}

It's necessary to simply the valve appropriately and add a large container at the inlet end of the valve to replace the piping system. The entire fluid-structure coupling model is shown in Figure 1. The gray part is the solid part and the blue part is the fluid part. After that, the mesh was divided and the independence of mesh was verified. Finally, boundary setting and simulation are carried out on the ANSYS Workbench platform.

*Corresponding author e-mail:yxhh@ecust.edu.cn 


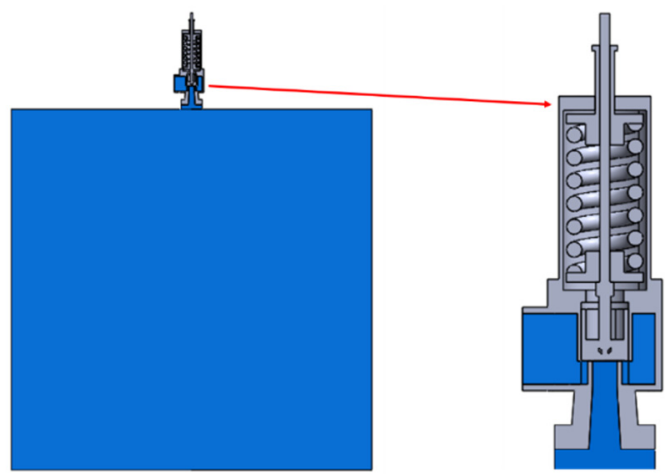

Fig. 1 FSI model of PRV

\section{Results and discussions}

\subsection{Simulation results without loading seismic waves} as a function of discharge time of PRV when no seismic wave is applied to the bottom of the valve seat. As shown in Figure 2, the maximum opening time of the valve is $3200 \mu \mathrm{s}$, the maximum opening height is $1.64 \mathrm{~mm}$, the return time is $8000 \mu \mathrm{s}$, and the return pressure is $0.59984 \mathrm{MPa}$.
Fig.2 is a graph showing the inlet vessel pressure and lift

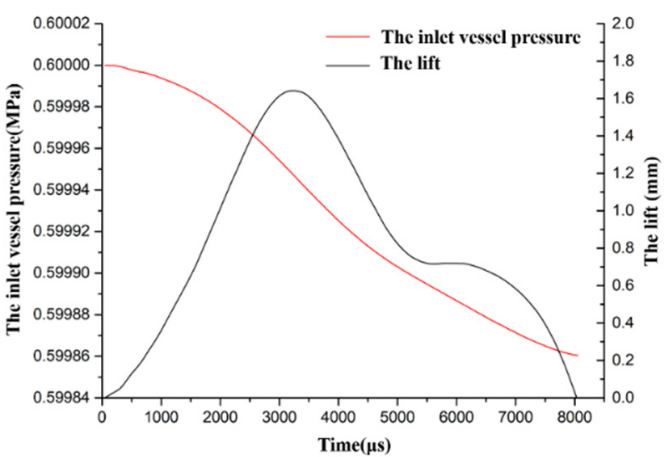

Fig. 2 The inlet vessel pressure and lift as a function of discharge time of PRV without loading seismic waves

Figure 3 shows the mesh displacement of the solid area in the $\mathrm{Y}$-axis direction. Figure 3(a) shows the mesh displacement of the solid area when before the valve opens and after the spring is pre-compressed. Figure 3(b) shows the mesh displacement of the solid area during stable discharge. It can be seen from the figure that before the valve is opened, the adjusting screw and the upper spring seat are compressed downward by $3 \mathrm{~mm}$, and the displacement of the spring is larger in the upper part while smaller in the lower part, until the displacement of the lower spring seat becomes $0 \mathrm{~mm}$. It can also be seen that in the process of stable discharge, the valve is displaced upward due to the impact of the fluid. The maximum total displacement is $1.7 \mathrm{~mm}$, which is consistent with the maximum opening height in the lift curve.

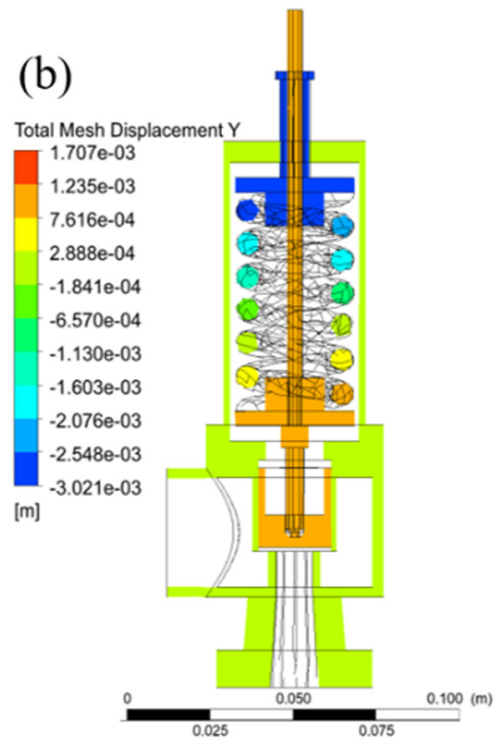

Fig.3 Mesh displacement in the $\mathrm{Y}$ direction of the solid area

Fig. 4 shows the stress distribution in the solid area, Fig. 4(a) shows the stress distribution in the solid area when before the valve is opened after the spring is precompressed, and Fig. 4(b) shows the stress distribution in the solid area during stable discharge. It can be seen from the figure that before the relief valve is discharged, the spring, disc, disc seat and guide sleeve have greater stress, and the stress on the spring is evenly distributed.
The maximum stress $(363 \mathrm{MPa})$ appears near the lower edge of the disc seat. After the relief valve stably discharges, the stress at the spring is uneven. The stress at the lower part of the spring is higher while the stress at the upper part is slightly lower. The maximum stress $(311 \mathrm{MPa})$ appears in the lower part of the spring. 

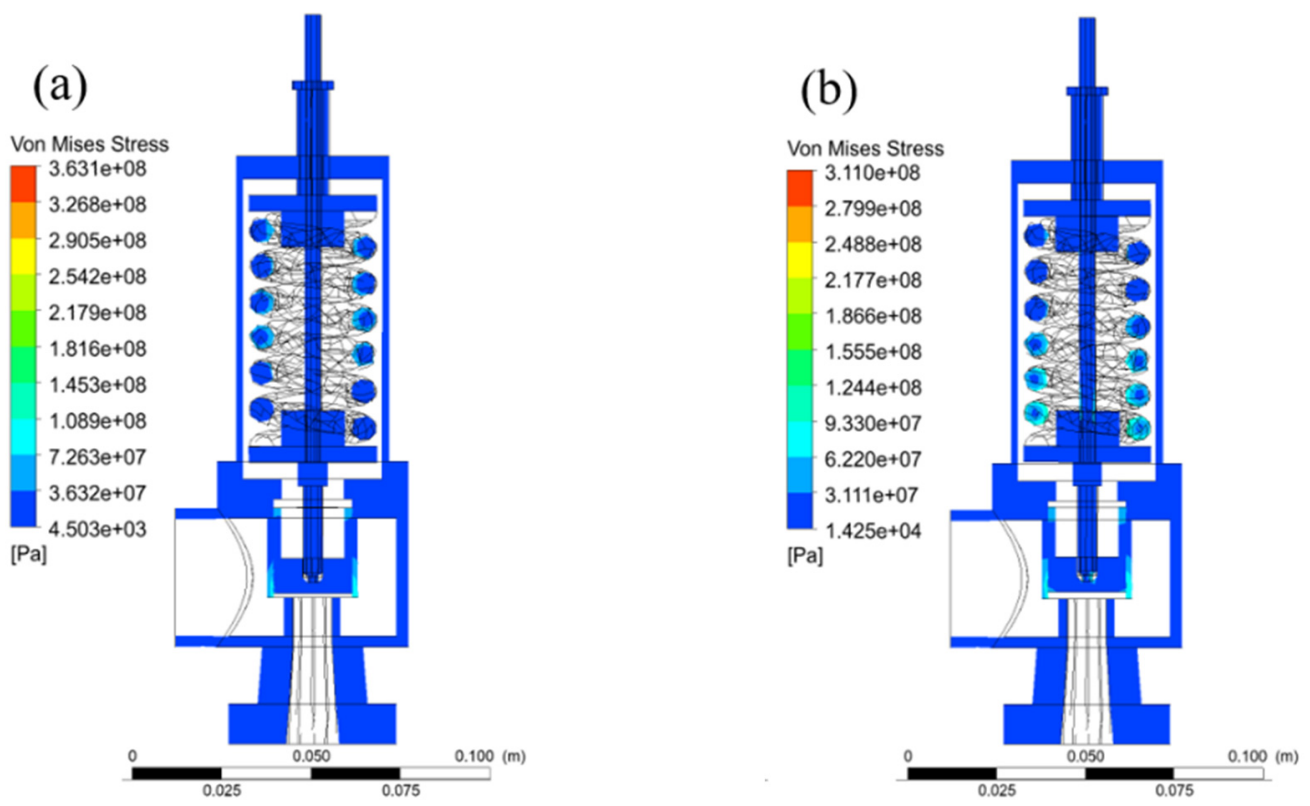

Fig.4 Stress distribution in the solid area

\subsection{Simulation results of loading seismic waves (frequency $16 \mathrm{~Hz}$, acceleration $6 \mathrm{~g}$ )}

A longitudinal seismic wave (frequency $16 \mathrm{~Hz}$, acceleration $6 \mathrm{~g}$ ) is loaded at the bottom of the valve seat. The seismic wave is loaded to the bottom of the valve seat in the form of displacement that formula is $\mathrm{x}=$ $0.0005938 * \sin (100.53 *$ time $)(\mathrm{m})$. Finally, Fig. 5 is a graph showing the inlet vessel pressure and lift as a function of discharge time of PRV.

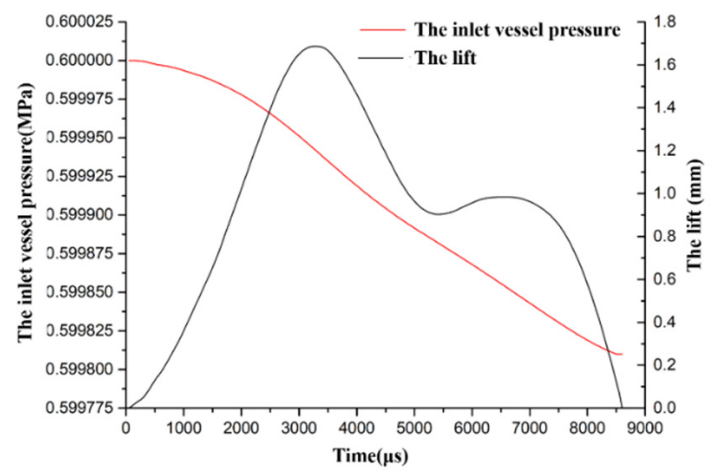

Fig. 5 The inlet vessel pressure and lift as a function of discharge time of PRV with loading seismic waves(frequency $16 \mathrm{~Hz}$, acceleration $6 \mathrm{~g}$ )

It can be seen from Figure 5 that the maximum opening time of the valve is $3300 \mu$ s, the maximum lift is
$1.69 \mathrm{~mm}$, the return time is $8500 \mu \mathrm{s}$, and the return pressure is $0.59981 \mathrm{MPa}$.

Figure 6 shows the mesh displacement of the solid area in the $\mathrm{Y}$-axis direction. Figure 6(a) shows the mesh displacement of the solid area when before the valve opens and after the spring is pre-compressed. Figure 6(b) shows the the mesh displacement solid area during stable discharge. It can be seen from the figure that before the valve is opened, the adjusting screw and the upper spring seat are compressed downward by $3 \mathrm{~mm}$, and the displacement of the spring is larger in the upper part while smaller in the lower part, until the displacement of the lower spring seat becomes $0 \mathrm{~mm}$. It can also be seen that in the process of stable discharge, the valve is displaced upward due to the impact of the fluid. The maximum total displacement is $1.7 \mathrm{~mm}$, which is consistent with the maximum opening height in the lift curve. 

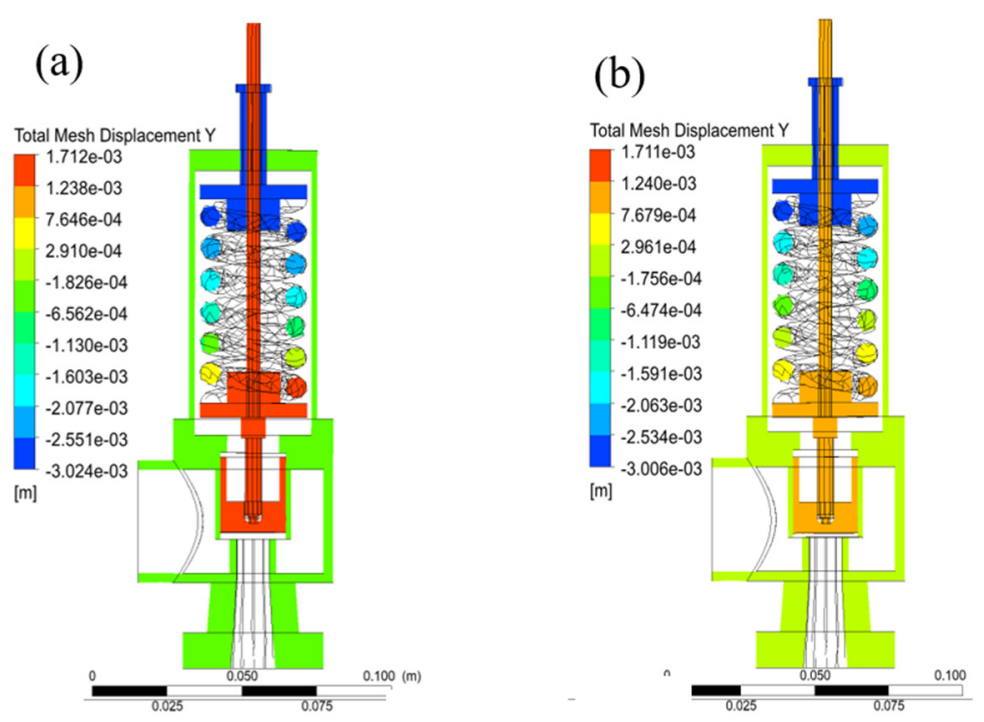

Fig.6 Mesh displacement in the Y direction of the solid area

Fig. 7 shows the stress distribution in the solid area.Fig. 7(a) shows the stress distribution in the solid area when before the valve is opened and after the spring is pre-compressed.Fig. 7(b) shows the stress distribution in the solid area during stable discharge. It can be seen from the figure that before the relief valve is discharged, the spring, disc, disc seat and guide sleeve have greater

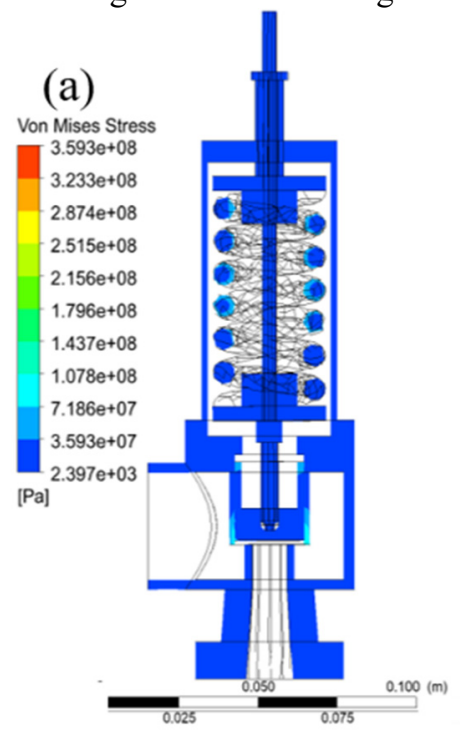

stress, and the stress on the spring is evenly distributed. The maximum stress (359MPa)appears near the lower edge of the disc seat. After the relief valve stably discharge, the stress at the spring is uneven. The stress at the lower part of the spring is higher while the stress at the upper part is slightly lower. The maximum stress $(329 \mathrm{MPa})$ appears in the lower part of the spring.

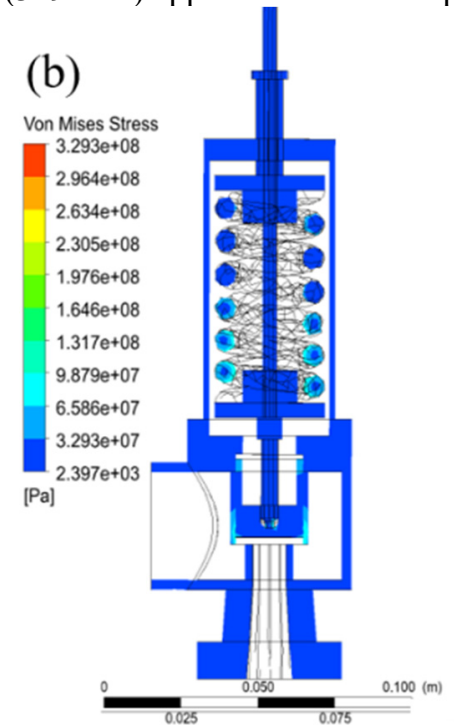

Fig.7 Stress distribution in the solid area

\subsection{Simulation results of loading seismic waves (frequency $32 \mathrm{~Hz}$, acceleration $6 \mathrm{~g}$ )}

A longitudinal seismic wave (frequency $32 \mathrm{~Hz}$, acceleration $6 \mathrm{~g}$ ) is loaded at the bottom of the valve seat. The seismic wave is loaded to the bottom of the valve seat in the form of displacement that formula is $\mathrm{x}=-\mathrm{x}=-$ $0.000148 * \sin (201.06 *$ time $)(\mathrm{m})$. Finally, Fig. 8 is a graph showing the inlet vessel pressure and lift as a function of discharge time of PRV.

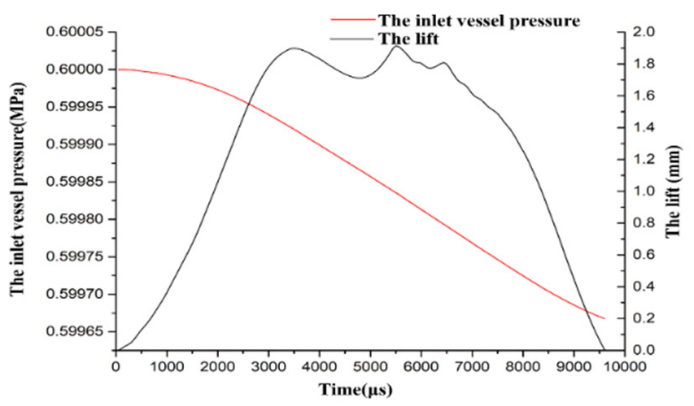

Fig. 8 The inlet vessel pressure and lift as a function of discharge time of PRV with loading seismic waves(frequency $32 \mathrm{~Hz}$, acceleration $6 \mathrm{~g}$ )

It can be seen from Fig. 8 that the maximum opening time of the valve is $5500 \mu \mathrm{s}$, the maximum lift is $1.91 \mathrm{~mm}$, 
the re-seat time is $9600 \mu \mathrm{s}$, and the re-seat pressure is $0.59967 \mathrm{MPa}$.

Figure 9 shows the mesh displacement of the solid area in the Y-axis direction. Figure 9(a) shows the mesh displacement of the solid area when before the valve opens and after the spring is pre-compressed. Figure 9(b) shows the the mesh displacement solid area during stable discharge. It can be seen from the figure that before the valve is opened, the adjusting screw and the upper spring

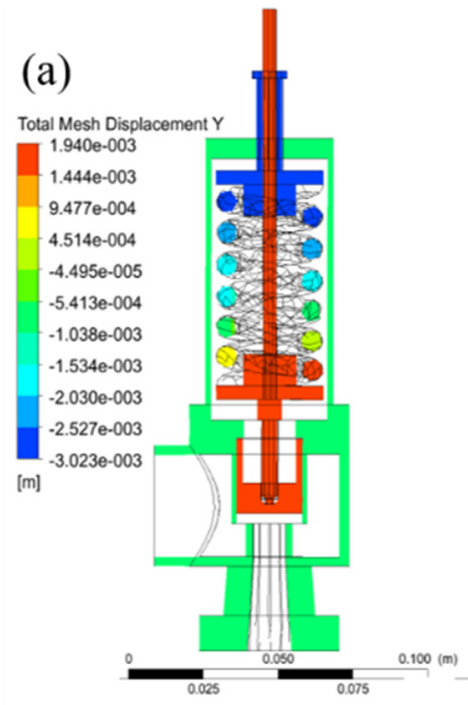

Fig.9 Mesh displacement in the Y direction of the solid area

Fig.10 shows the stress distribution in the solid area.Fig.10(a) shows the stress distribution in the solid area when before the valve is opened and after the spring is pre-compressed.Fig.10(b) shows the stress distribution in the solid area during stable discharge. It can be seen from the figure that before the relief valve is discharged, the spring, disc, disc seat and guide sleeve have greater

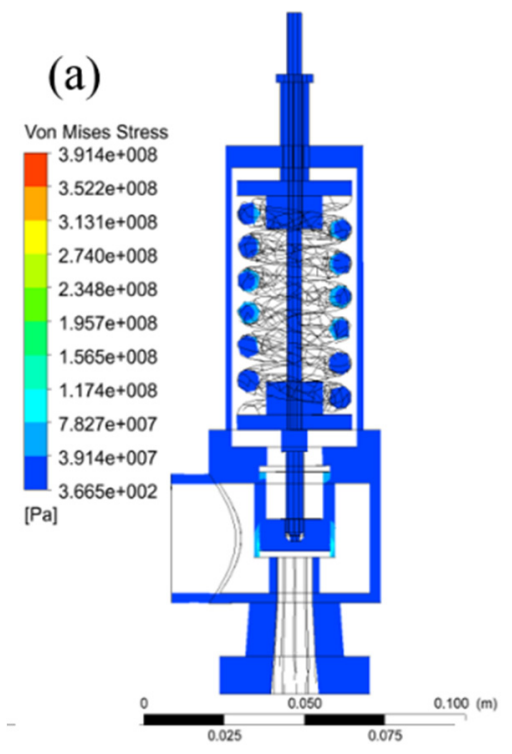

seat are compressed downward by $3 \mathrm{~mm}$, and the displacement of the spring is larger in the upper part while smaller in the lower part, until the displacement of the lower spring seat becomes $0 \mathrm{~mm}$. It can also be seen that in the process of stable discharge, the valve is displaced upward due to the impact of the fluid. The maximum total displacement is $1.9 \mathrm{~mm}$, which is consistent with the maximum opening height in the lift curve.

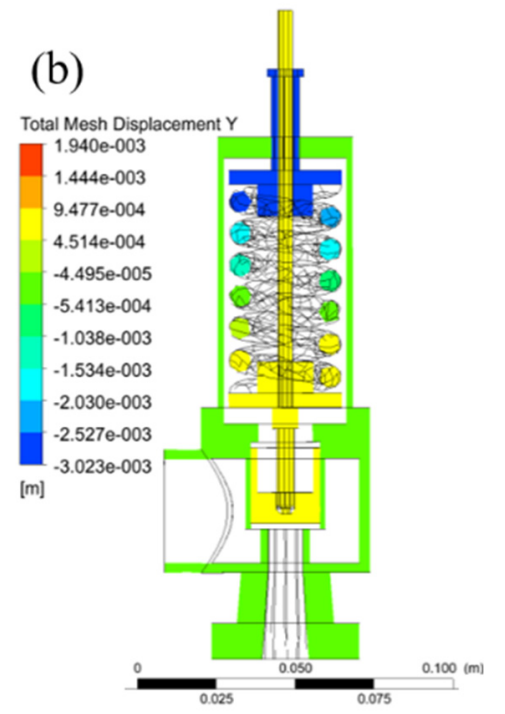

stress, and the stress on the spring is evenly distributed. The maximum stress (391MPa)appears near the lower edge of the disc seat. After the relief valve stably discharge, the stress at the spring is uneven. The stress at the lower part of the spring is higher while the stress at the upper part is slightly lower. The maximum stress $(391 \mathrm{MPa})$ appears in the lower part of the spring.

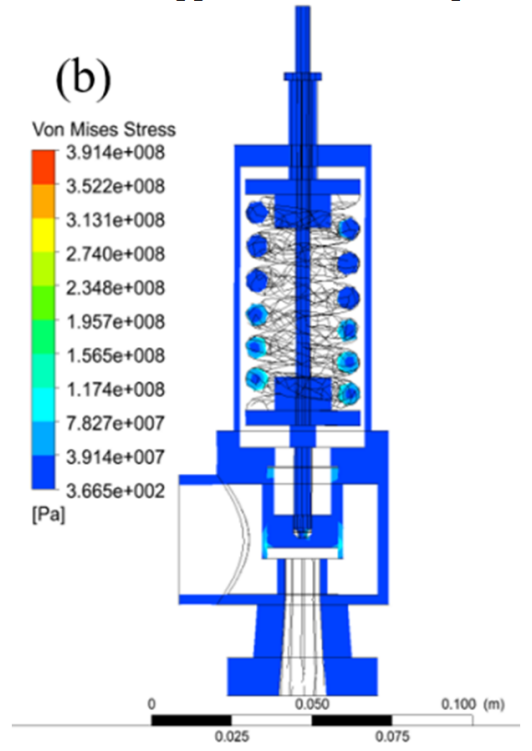

Fig.10 Stress distribution in the solid area

\subsection{Comparison of simulation results}

Fig.11(a) shows that comparison of the lift curves under seismic and non-seismic conditions, while Fig.11(b) shows comparison of the inlet pressure curves of the relief valve under seismic and non-seismic conditions. It can be seen that with the loading of the longitudinal sine wave, the lift of the relief valve increases slightly. The $1.7 \mathrm{~mm}$ under non-seismic conditions rises to $1.9 \mathrm{~mm}$ under seismic conditions with frequency $32 \mathrm{~Hz}$ and acceleration $6 \mathrm{~g}$. The change that the return pressure of 
the relief valve changes slightly from $0.59990 \mathrm{MPa}$ to

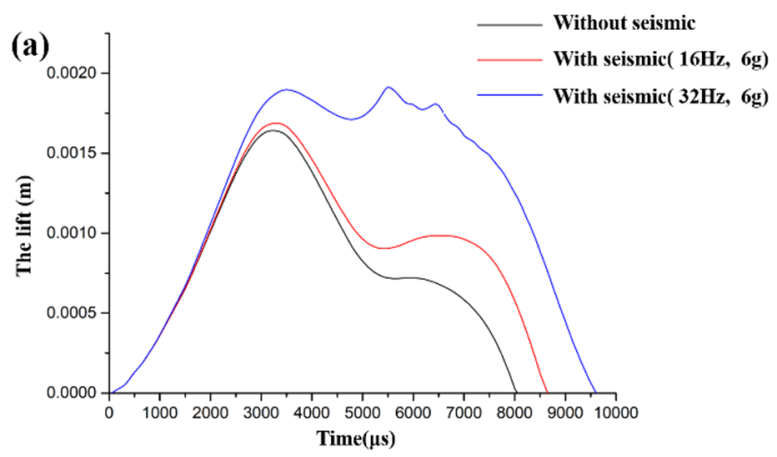

Fig.11 Comparison of lift(a) and inlet pressure(b)

\section{Action performance test of relief valve under seismic conditions}

According to the actual working conditions of the valve, a test platform capable of simulating seismic conditions was established. The action performance test results of the relief valve without seismic conditions are shown in Fig.12(a). The set pressure is $0.58 \mathrm{MPa}$ and the return pressure is $0.48 \mathrm{MPa}$. The action performance test results of the relief valve under longitudinal loading of a seismic wave (frequency $32 \mathrm{~Hz}$, acceleration $6 \mathrm{~g}$ ) are shown in Fig.12(b). The set pressure is $0.60 \mathrm{MPa}$ and the return pressure is $0.48 \mathrm{MPa}$. It can be seen that under the condition of longitudinal seismic wave, the return pressure of the relief valve is almost constant, which is consistent with the result trend of Fig.11. Due to the longitudinal loading of the seismic wave, the setting

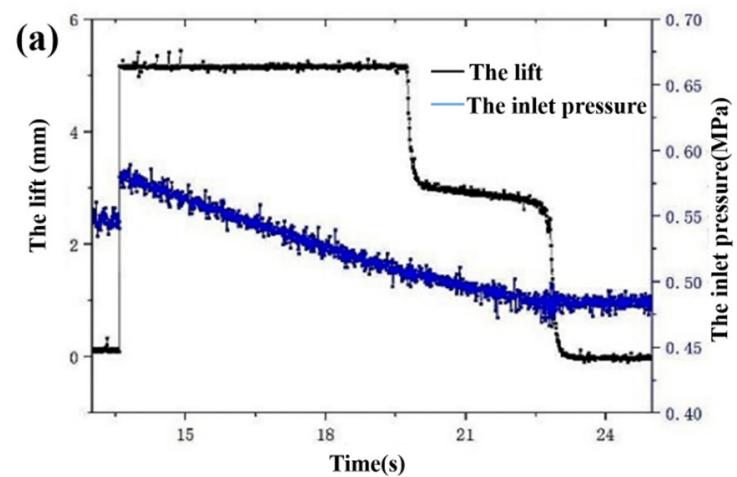

(b)

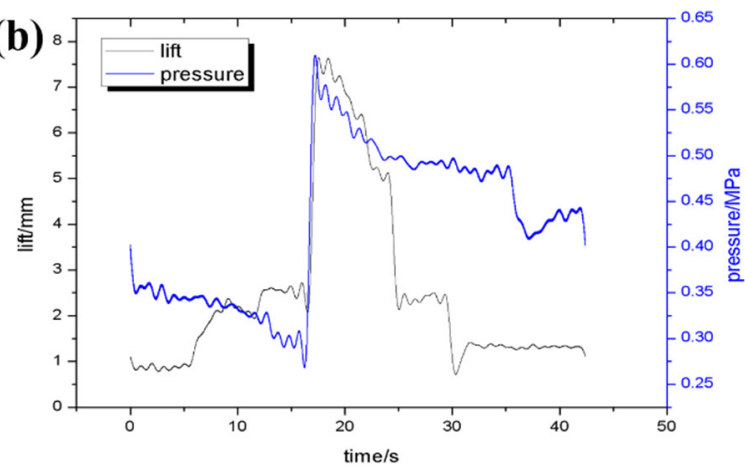

Fig.12 Operation performance test results without wave(a) and with loading longitudinal sine wave(b) on relief valve

\section{Conclusion}

Using dynamic grid, watershed block, external virtual large container and other simulation technologies, the simplified pressure stabilizer relief valve model is used to conduct two-way transient fluid-solid coupling dynamic simulation. The contact pairs between the fluid field and the solid coupling surface and the solid parts are accurately set, and the dynamic characteristics of the fluid area and the stress and strain of the solid area are solved simultaneously. The action of the longitudinal seismic wave on the relief valve is preliminarily explored by loading the sine wave. The results show that the effect of longitudinal seismic load on the return pressure of the relief valve is negligible.
$0.59965 \mathrm{MPa}$ can be ignored.

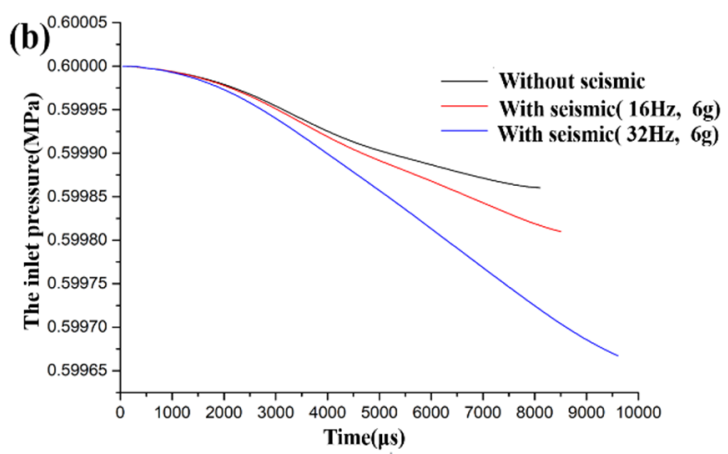

(b) curves for seismic and non-seismic conditions

pressure of the relief valve increases slightly. It is because that the longitudinal vibration causes the sealing performance of the relief valve to decrease, and the relief valve leaks before the jump, causing the setting pressure of the relief valve to increase slightly. The reseat pressure value obtained in the test is slightly lower than the simulated reseat pressure value $(0.5998 \mathrm{MPa})$. These differences are caused by the simplified structure. Although there are differences in numerical values, it is very accurate that the longitudinal seismic wave predicted by the two-way fluid-solid coupling simulation has a negligible effect on the return pressure of the relief valve. In the later stage, the action performance characteristics of the pressure stabilizer relief valve under more complicated seismic conditions can be further studied through the two-way fluid-solid coupling.

\section{References}

1. Guo Changsheng.Moving Mesh Based Flow Field Simulation of Pressure Regulating Valve and Spool Motion Analysis[D]. Chongqing University,2013

2. Deng J, Shao X M, Fu X, et al. Evaluation of the viscous heating induced jam fault of valve spool by fluid-structure coupled simulations[J]. Energy Conversion \& Management, 2009, 50(4):947-954.

3. ZHOU Zhenfeng.Stress Study on Flexure Tube for Nozzle-Flapper Electrohydraulic Servovalve Based on Two-way Fluid-Structure Coupling Simulation Analysis[J]. MACHINE TOOL \& HYDRAULICS,2017,45(11):161-165 
4. Sang-Uk Han, Dae-Gyun Ahn, Myeong-Gon Lee, et al. Structural Safety Analysis Based on Seismic Service Conditions for Butterfly Valves in a Nuclear Power Plant [J]. The Scientific World Journal, 2014, 1:1-9.

5. ZhaoYun,, HuGuangzhong, WenHuabin, ZouLiang, JiBokai. Study on Fluid-solid Coupling Characteristics during Spherical Check Valve Closing Process[J].Fluid Machinery, 2019, 47(5): 28-32

6. Koji Nishino, Yoshitaka Tsutsumi, Kazuyoshi Yonekura et al. Seismic test results of the main steam safety relief valve for japanens boiling water reactor nuclear power plants $[\mathrm{J}]$. Proceedings of the ASME 2017 Pressure Vessels and Piping Conference, 2017, 1-5.

7. Chen Dexiang, Liu Hao, Geng Maofei, Wang Le, Zhang Chengyan. Fluid Structure Interaction Analysis of Reed Valve for Micro-compressor[J]. Fluid Machinery, 2020, 48(6): 20-24

8. Liu Fusheng, Wang Hong, Zhang Qinzhao. Experimental Research on the Flow Resistance Characteristics of the Tri-eccentric Butterfly[J]. Fluid Machinery, 2020, 48(3): 12-16 